\title{
USO DEL HUMOR COMO MÉTODO DE INTERVENCIÓN TERAPÉUTICA EN MENORES AFECTADOS POR PROCESOS ONCOLÓGICOS
}

\section{Use of humour as a method of therapeutic intervention with children affected by oncological processes}

\author{
María Fernández Hawrylak \\ Correo-e: mfernandez@ubu.es \\ Davinia Heras Sevilla \\ Correo-e: dheras@ubu.es \\ Amaya Cepa Serrano \\ Correo-e: acepa@ubu.es \\ Julia Bastida Susinos \\ Facultad de Educación. Departamento de Ciencias de la Educación. \\ Universidad de Burgos \\ Correo-e: jbsoo46@alu.ubu.es
}

Recepción: 9 de octubre de 2016

Envío a informantes: is de noviembre de 2016

Aceptación definitiva: 20 de mayo de 2017

Resumen: La presencia de los payasos (clowns) en los hospitales, especialmente en el ámbito pediátrico, comienza a ser habitual, constituyendo una medida recurrente. Son muchos los países donde los niños reciben la actuación de los payasos durante su hospitalización. Sin embargo, todavía son pocos los estudios que se han dedicado a analizar en profundidad los beneficios que aportan estas intervenciones con los menores ingresados en su adaptación al contexto hospitalario y en la aceptación de la enfermedad. El objetivo de la investigación que se presenta es comprobar si el uso del humor como estrategia activa de afrontamiento de la enfermedad es eficaz en los niños oncológicos y sus familias. Se utilizó el estudio de casos como metodología de investigación. La muestra estuvo compuesta por cuatro niños/as afectados por enfermedades oncológicas, con edades comprendidas entre los 5 y 8 años, ingresados en el Servicio de Pediatría del Hospital Universitario de Burgos, alumnos del Aula Hospitalaria, y sus familias. Los instrumentos utilizados fueron una entrevista semiestructurada realizada a las familias, 
y un cuestionario diseñado ad hoc tipo Likert para los niños. Se informatizaron y codificaron los datos procedentes de los cuestionarios. Asimismo, se realizó una codificación y categorización de la información procedente de las entrevistas atendiendo a criterios temáticos y gramaticales. Los resultados obtenidos demuestran que el humor mejora la estancia del niño y de sus familiares en el hospital, haciendo que se distraigan y olviden temporalmente la enfermedad. Como conclusión cabría destacar que el empleo de humor no soluciona ningún problema en sí mismo, pero puede cambiar la forma de abordar la enfermedad y, por tanto, constituye una estrategia activa de afrontamiento.

Palabras Clave: humor; enfermedades oncológicas; estrategias de afrontamiento; payaso de hospital; aula hospitalaria; estudio de caso.

Aвstract: Clowns in hospitals begin to be common, in particular in the paediatric sphere, becoming a recurring action. Although there are many countries in the world where children receive the performance of clowns during their hospital stay, yet few studies have been devoted to make a deep analysis of the effectiveness of hospital clowns interventions in the adaptation to hospitalization and in the acceptance of the disease. This research aims to check if the use of humour as an active disease coping strategy is effective in children with cancer and their families. Case study was used as research method. The sample consisted of four children affected with oncological diseases, aged between 5 and 8 , hospitalized in the paediatric ward of the University Hospital of Burgos and attending the Hospital School, and their families. The instruments used were semi-structured interviews with the families, and an ad hoc designed Likert questionnaire for the children. Data from questionnaires were coded and analysed. The information from the interviews was coded and categorized according to thematic and grammatical criteria. The outcomes show that humour improves the hospital stay of the children and their relatives, gets them distracted and makes them forget their disease for a while. It would be highlighted, in conclusion, that the use of humour does not solve any problem itself, but could change the way of addressing the disease, therefore it is an active form of coping.

KEY WORDs: humour; oncological diseases; coping strategies; hospital clown; hospital school; case study.

\section{Introducción}

$\mathrm{E}$

L HUMOR HA SIDO CONCEPTUALIZADO DE DIFERENTES MANERAS: como respuesta, proceso cognitivo, habilidad, capacidad, rasgo de personalidad, etc. Desde una perspectiva teórica se puede entender el humor como un constructo multidimensional y complejo; implica elementos sensoriales, emocionales, cognitivos y expresivos que interrelacionan entre sí. Dada esta naturaleza heterogénea, existe una amplia variedad de definiciones, siendo extremadamente difícil encontrar una descripción universal y perfectamente satisfactoria (Ruch, I998).

Dicho constructo podría encuadrarse dentro de considerandos muy familiares a la vez que desconocidos. Familiares porque está presente en todos los seres humanos a pesar de que puedan existir diferencias culturales (Martin, Puhlik-Doris, Larsen, Gray y Weir, 2003; Martin, 2004); precisamente, la sonrisa aparece durante el primer mes de vida, y la risa ocurre alrededor del cuarto mes (Martin, 200o). Desconocidos, 
USO DEL HUMOR COMO MÉTODO DE INTERVENCIÓN TERAPÉUTICA

EN MENORES AFECTADOS POR PROCESOS ONCOLÓGICOS

M. FERNÁNDEZ HAWRYLAK, D. HERAS SEVILLA, A. CEPA SERRANO Y J. BASTIDA SUSINOS

porque, a pesar de venirse estudiando desde hace algunas décadas, el debate sobre qué es el humor, para qué sirve, cómo se produce o cómo se miden sus beneficios continúa rodeado de diversos desacuerdos (Klein, 2003; Norrick, 2003; Perlmutter, 2000), lo cual implica que deba seguirse investigando.

La risa es la exhibición externa de humor. Aunque puede haber humor sin risa o risa sin humor, ambos mantienen una estrecha relación. La risa es una manifestación biológica producida por el organismo como respuesta a determinados estímulos, y considerada básicamente una reacción a momentos o situaciones de humor como expresión externa de diversión y relacionada con alegría, motivada por un estímulo cómico y que suele producirse en relación con los otros dentro de la comunicación (Provine, 2004).

En el Diccionario de uso del español de Moliner (200I) se define humor como «el estado de ánimo de una persona, habitual o circunstancial, que le predispone a estar contenta y mostrarse amable, o, por el contrario, a estar insatisfecha y mostrarse poco amable», es decir, como una actitud subjetiva. Autores como Carbelo y Jáuregui (2006: 19) consideran el humor como «cualquier estímulo que pueda provocar la risa de un participante: juegos, bromas, chistes, viñetas, situaciones embarazosas, incongruencias, inocentadas, cosquillas...». Se podría decir que el humor, como generador de risas, se convierte en una estrategia para comunicarse y enfrentarse a diversas situaciones de la vida cotidiana, constituyendo una actitud ante la vida. Puede ser, por tanto, un recurso para enfrentarse a circunstancias valoradas como desagradables o negativas por la persona, favoreciendo procesos de regulación emocional en beneficio del bienestar personal y la adaptación al entorno social.

El humor comienza a ser objeto de estudio en investigaciones desde diversas disciplinas. Desde la perspectiva de la piscología positiva, el humor es considerado un rasgo estable de la personalidad y un constructo multidimensional en el que convergen tres factores: uno cognitivo, que implicaría el análisis y la interpretación de lo que sucede; otro afectivo, que sería la manera de sentirse alegre ante las circunstancias de la vida; y uno comportamental, que consistiría en la manifestación del sentido del humor (Fernández-Abascal, 2009).

Son muchos los estudios científicos que examinan los beneficios de la risa (Balick y Lee, 2003; Berk, 200I; Camuñas, 2009; Mora y Quintana, 20Io; Sultanoff, 1999), siendo algunos de ellos la reducción de la ansiedad, del estrés, de la depresión, de la tensión, de la soledad; el aumento de la autoestima, de la esperanza, la aceptación, la comprensión, la alegría, la tranquilidad y/o la seguridad. Es decir, cambios emocionales positivos.

Igualmente, el humor puede mejorar aspectos fisiológicos, como la tolerancia al dolor, la mejora del sistema cardiovascular y especialmente la del sistema inmunitario. Reírse produce una serie de efectos sistémicos que son beneficiosos para el cuerpo, tales como relajación muscular, disminución de la glucemia, incremento de la saturación en sangre y movilización de secreciones respiratorias (Sánchez, Gutiérrez, Santacruz, Romero y Ospina, 2009).

Desde una perspectiva social, también son considerables los beneficios. Se han encontrado mejoras en la comunicación, en el orden y en la armonía social. Además, el humor es un medio adecuado para fortalecer relaciones, al desencadenar sentimientos positivos e impulsar la conexión emocional. Compartir la risa permite el establecimiento de relaciones interpersonales sanas (Carbelo, 2005). Incluso puede llegar a 
ser un tratamiento complementario para mantener el bienestar físico y mental (Ruiz, Rojo, Ferrer, Jiménez y Ballesteros, 2005).

Podrían entonces reconocerse los efectos de la risa desde el punto de vista fisiológico, psicológico y social. Sin embargo, no todas las investigaciones encuentran asociaciones positivas entre el sentido del humor y medidas de salud, ajuste o bienestar (Capps, 2006; Erickson, y Feldstein, 2007; Kuiper, Grimshaw, Leite y Lirsh, 2004). Estos estudios llevan a la necesidad de una conceptualización más elaborada del humor y a considerar que éste también tiene componentes negativos y no sólo positivos. En este sentido cabe destacar la tipología descrita por Martin (200I), para quien existen fundamentalmente cuatro tipos de humor: afiliativo, orientado al mejoramiento personal, agresivo y de descalificación personal. Esta investigación se centra en las dos primeras clases de humor, dado su carácter positivo e incluso terapéutico. Por un lado, se estudia el humor social entendido como aquel que involucra bromear con los demás, contar historias divertidas y reír con otros en un intento por amortiguar la tensión interpersonal. Y, por otro lado, se analiza el humor que acrecienta el self, es decir, aquel que implica una visión humorística y una tendencia a mantener el humor cuando se experimentan circunstancias adversas.

Autores como Martin (2000) y Perlmutter (2000), entre otros, han realizado un intento por englobar los postulados clásicos para la configuración del marco conceptual del humor. Básicamente, se podrían distinguir tres categorías teóricas generales: teorías de superioridad o de denigración, donde el humor aparece en la percepción de los demás en cuanto a sus debilidades y/o deficiencias (Ford y Ferguson, 2004; Gruner, I997; Joubert, I579/2002; Perlmutter, 2000); teorías de la incongruencia, basadas en la ocurrencia de un suceso inesperado que sucede sin que sea lo lógico (Attardo, Hempelmann y Di Maio, 2002; Carbelo, 2005; Forabosco, 1992; Roeckelein, 2002; Wycoff y Pryor, 2003); y teorías del alivio/descarga, que ven el humor como un mecanismo de defensa ante la adversidad (Billing, 2002; Fry, 2002).

No obstante, es complejo enmarcar esta investigación exclusivamente en alguna de las teorías sobre el humor. La existencia de una gran variedad de las mismas hace difícil su clasificación (Roeckelein, 2002). Posiblemente, las más afines sean las teorías de la incongruencia, vinculadas a situaciones ambiguas, inciertas, que provocan una respuesta de humor casi siempre ocasionada por una paradoja, y asociadas a la emoción de sorpresa, así como a las teorías de alivio/descarga, que postulan que a través de chistes y bromas pueden manifestarse los deseos más reprimidos.

Cuando se goza de buen humor se aprende a mirar los problemas de una forma diferente, de manera más relajada, y se encuentran soluciones más creativas y flexibles más rápidamente. Aunque el sentido del humor no solucione ningún problema por sí mismo puede cambiar la forma de abordarlo, o, en el caso que nos concierne, de hacer frente a la propia enfermedad (Camuñas, 2009), esto es, usando el humor como una estrategia positiva de afrontamiento para regular las emociones negativas y afrontarlas, de forma que la persona no se dañe a sí misma ni a otros.

El humor y la risa sirven para olvidar temporalmente los problemas. Dentro de los profesionales que son expertos en producir una sonrisa en el rostro humano se encuentran los payasos. Clowns artísticos, que tratan de generar sensaciones esperanzadoras y positivas por medio del tratamiento cómico de situaciones de la vida cotidiana. Se puede hablar de payasos sociales, que desarrollan ayuda humanitaria a través de formación y/o espectáculos para la mejora de las condiciones de vida, y de los 
USO DEL HUMOR COMO MÉTODO DE INTERVENCIÓN TERAPÉUTICA

EN MENORES AFECTADOS POR PROCESOS ONCOLÓGICOS

M. FERNÁNDEZ HAWRYLAK, D. HERAS SEVILLA, A. CEPA SERRANO Y J. BASTIDA SUSINOS

clowns terapéuticos, que promueven la salud y el bienestar mediante la estimulación del descubrimiento lúdico, la expresión o apreciación de lo absurdo de las situaciones de la vida. Dentro de estos últimos, se encuentran aquellos que promueven la salud en los hospitales (Baliari y Rosado, 20I0; Camuñas, 2009; Koller y Gryski, 2008), los hospital clowns. El papel del payaso en los hospitales es hacer reír aportando a la vez optimismo y esperanza dentro de una situación dramática como puede llegar a ser la enfermedad. No significa acudir a sanar a un paciente con una nariz de payaso y una vestimenta peculiar. Se trata de utilizar estrategias de intervención como la risa, para reducir los efectos negativos causados por la enfermedad, tales como ansiedad, tristeza, baja autoestima u otros que afectan al desarrollo intelectual, afectivo y social.

La salud, como responsabilidad individual y colectiva, debe ser atendida en condiciones óptimas y desde un estado mental positivo, a fin de procurar una mayor adaptación a la enfermedad. El humor puede brindar la atmósfera adecuada, posibilitando el efectivo abordaje de la enfermedad. Es un medio para aliviar el padecimiento y distraer a los participantes de las rutinas y acontecimientos del ámbito hospitalario, situando a los pacientes en un clima más receptivo para afrontar los déficits y cuidar la salud. En esta línea, en 197I el médico Patch Adams fundó el Instituto Gesundheit como respuesta al cuidado de la salud en Estados Unidos. El objetivo de Adams era llevar diversión, felicidad y amistad del servicio a la práctica médica (Instituto Gesundheit, s. f.).

El primer programa de payasos de hospital fue fundado por Michael Christensen en Nueva York en el año I986 (De la Gándara, 2013). Su fundador afirmaba que «los payasos no pertenecen a los hospitales, los niños tampoco». Aun así, los payasos de hospital se han expandido por el mundo formando parte del ámbito sanitario, buscando que tanto los pacientes como sus familiares tengan una estancia hospitalaria y unos tratamientos más amenos (Baliari y Rosado, 20IO). Todos los grupos de clowns se rigen por el Código Deontológico de Clowns (Christian, Ramos, Susanibar y Balarezo, 2004).

En los últimos años existe un fuerte incremento de la presencia de los payasos en los hospitales (Gutiérrez et al., 2008), especialmente en Pediatría. Son muchos los países del mundo donde los niños reciben ya la actuación de los payasos durante su hospitalización (Koller y Gryski, 2008). Pero también hay experiencias con adultos en diversos servicios hospitalarios donde se emplea el humor y la risa como terapias no farmacológicas. Sin embargo, son pocos los estudios empíricos que se han dedicado a hacer un análisis en profundidad sobre la eficacia en las intervenciones de los payasos de hospital, aunque sí podrían citarse algunos (Gutiérrez et al., 2008; Meisel et al., 2009).

En España empiezan a proliferar algunas iniciativas que tienen como tema central el humor, como por ejemplo «La Sonrisa Médica», «Theodora», «Pupaclowns», «Payasospital», «Salut i Clowns», etc. Los payasos de hospital que visitan el Hospital Universitario de Burgos (HUBU), contexto donde se enmarca este trabajo, se denominan «Terapiclowns» y lo componen cinco artistas. El objetivo principal de Terapiclowns es «aplicar la filosofía y técnica clown en diferentes áreas hospitalarias y poner en práctica las propiedades terapéuticas de la risa» (Benito, y García, 20I3: 8). Las intervenciones de estos profesionales son personalizadas, siempre son actuaciones de dos personas caracterizadas, y se realizan dentro de las habitaciones del hospital donde intentan adaptarse al estado anímico del niño y a su enfermedad. Organizan visitas 
mensuales y están presentes los niños, sus familiares y el equipo médico-sanitario, que también se benefician de la actividad. Es un medio para conseguir el mayor número de sonrisas de los pequeños pacientes que están hospitalizados.

El humor y la risa se vienen utilizando con frecuencia en los hospitales como estrategias conductuales para reducir el estrés, el miedo y la ansiedad. La intervención de los payasos de hospital es uno de los programas que emplea el humor para disminuir el malestar psicológico infantil del niño en el ámbito hospitalario (Meisel et al., 2009). Entramos aquí en materia de estrategias de afrontamiento, las cuales forman un conjunto de procesos cognitivos, afectivos y comportamentales que utilizan las personas para solucionar conflictos de la vida diaria o para convivir con ellos.

De las revisiones realizadas por Espada, Grau y Fortes (20I0) pueden extraerse dos tipos de estrategias de intervención: activas y pasivas. Las estrategias activas pueden ir dirigidas a la emoción (el participante se enfrenta al problema y va regulando sus emociones de modo que las consecuencias aversivas del problema no le afecten emocionalmente) o al problema (el participante se enfrenta al problema regulando su comportamiento para disminuir las consecuencias aversivas que éste le produce), como resolución de problemas, regulación afectiva, búsqueda de apoyo social, distracción, negociación, reestructuración, búsqueda de información, etc. Las estrategias pasivas hacen referencia a la ausencia de enfrentamiento o a las conductas de evasión y negación, tales como huida o evitación, aislamiento social, rumiación del pensamiento, oposición y confrontación, desesperación o abandono, etc.

Las personas con enfermedad y sus familias utilizan ambos tipos de estrategias, tanto activas como pasivas. No se puede identificar una única estrategia como la mejor para todas las situaciones; cada participante utilizará o combinará las que mejor y más efectivas le resulten en cada situación. El sentido del humor puede emplearse de manera eficaz para desorientar a los individuos, para alejarlos de las situaciones potencialmente hostiles y agresivas, por lo que es muy importante identificar estas situaciones (Torres, 2003). Por tanto, el humor puede ser utilizado como una estrategia activa de afrontamiento ante una enfermedad grave que suele ser vivida como una situación agresiva, y empleada por las personas resilientes.

La incidencia y la mortalidad por cáncer están aumentando rápidamente en todo el mundo, especialmente en los países de ingresos bajos y medios. En los países de ingresos altos, aproximadamente el $80 \%$ de los niños con cáncer sobreviven cinco años o más después del diagnóstico. Las tasas mundiales de incidencia oscilan entre 50 y 200 por cada millón de niños en las distintas partes del planeta (Forman et al., 20I4). El cáncer infantil presenta cada vez una mayor importancia en el ámbito de la Pediatría.

Como recoge la Federación Española de padres de niños con cáncer (FEPNC, 2OI4) en su página web, cada año se diagnostican en España cerca de 1400 nuevos casos de cáncer en niños entre o y I8 años. Es la primera causa de muerte por enfermedad hasta los I4 años. En general, los tipos más frecuentes de cáncer en la infancia se pueden dividir en dos grandes grupos: las enfermedades hematológicas (leucemias y linfomas) y los tumores sólidos, siendo la leucemia el tipo de cáncer más frecuente en los niños ( $25 \%)$, seguido de los tumores del sistema nervioso central (19,6\%) y los linfomas (13,6\%), según el Registro Nacional de Tumores Infantiles (AECC, s. f.; FEPNC, 20I4).

La adaptación al cáncer es un proceso continuo en el cual el niño y su familia aprenden a regularse emocionalmente, solucionar problemas relacionados con el cáncer y controlar acontecimientos vinculados con la enfermedad. Afrontan muchos 
USO DEL HUMOR COMO MÉTODO DE INTERVENCIÓN TERAPÉUTICA

EN MENORES AFECTADOS POR PROCESOS ONCOLÓGICOS

M. FERNÁNDEZ HAWRYLAK, D. HERAS SEVILLA, A. CEPA SERRANO Y J. BASTIDA SUSINOS

desafíos y momentos de crisis, entre ellos, escuchar el diagnóstico, asimilar los cambios o hacer frente a los tratamientos (FEPNC, 2007).

La risa genera cambios positivos en el comportamiento emocional de los niños, de los padres y de los profesionales que los atienden (Battrick, Glasper, Prudhoe y Weaver, 2007). Junto con el juego, representa una de las técnicas más empleadas para ayudar al niño con cáncer y a sus familias a afrontar la enfermedad, dado que permite la liberación de tensiones físicas y emocionales, produce un cambio de actitud, el desarrollo de comportamientos positivos y de cumplimiento de las prescripciones médicas, mejorando la calidad de vida del niño (Barkmann, Siem, Wessolowski y Schulte-Markwort, 2013; Linge, 2013; Meisel et al., 2009).

Como se ha indicado, actualmente uno de los recursos utilizados tanto España como en otros países para reducir la ansiedad infantil y el olvido temporal de la enfermedad en el contexto sanitario son los payasos de hospital. Gracias a estos profesionales, muchos pequeños reducen sus emociones negativas durante todo el proceso patológico, fomentando además la relación con los profesionales sanitarios y desdramatizando algunas de las vivencias que les rodean. Pero todavía siguen siendo escasos los trabajos que inciden en la eficacia de sus actuaciones.

El presente estudio tiene como objetivo comprobar si el uso del humor como técnica activa de afrontamiento de la enfermedad es eficaz en niños oncológicos de larga duración y sus familias. Para ello se realizó un estudio de casos con el fin de conocer los efectos del humor a través de los payasos del hospital en el contexto del Aula Hospitalaria donde participan los menores afectados, sus familias, los maestros del aula en cuestión, el personal médico-sanitario y los autores.

De acuerdo también a la revisión teórica expuesta, el estudio parte de las siguientes preguntas de investigación: ¿El uso del humor mejora el bienestar en los menores hospitalizados con enfermedades oncológicas? ¿Es beneficioso trabajar el humor con las familias de estos menores? ¿Son efectivos los payasos de hospital en la estancia de los menores hospitalizados? ¿Los payasos de hospital ayudan a afrontar la enfermedad?

\section{Método}

\section{I. Participantes}

La muestra está compuesta por cuatro menores que presentan enfermedades oncológicas, tres niñas y un niño con edades comprendidas entre los 5 y los 8 años, con una edad media de $7.25(D T=$ I.5). Se trata de alumnado escolarizado en Educación Infantil (EI) y en Educación Primaria Obligatoria (EPO). Todos ellos han sido hospitalizados durante una semana o más, o se encuentran en el segundo ingreso en el HUBU.

\subsection{Instrumentos}

Se utilizan los siguientes instrumentos:

- Cuestionario breve de cinco ítems sobre la percepción infantil de los payasos en el hospital elaborado ex profeso con una escala tipo Likert [«Nunca» (I), «pocas veces» (2), «algunas veces» (3), «la mayoría de las veces» (4) y «siempre» 
(5)]. Aunque el cuestionario es una técnica clásica de recogida de datos en la investigación cuantitativa, también puede prestar un importante servicio en la investigación cualitativa siempre que se respeten algunas exigencias fundamentales (Rodríguez, Gil y García, I999) que se han cumplido.

- Entrevista semiestructurada que consta de cuatro preguntas abiertas dirigidas a conocer la opinión de los padres de los menores en torno a cómo influyen los payasos de hospital en la estancia del niño hospitalizado. Las preguntas tienen estrecha relación con las del cuestionario, pero tratan de averiguar las relaciones de los niños con los clowns desde la perspectiva del adulto.

\subsection{Procedimiento}

El estudio se enmarca dentro del paradigma sociocrítico, puesto que se intenta comprender e interpretar un fenómeno dentro de una situación real. Se elige un estudio cualitativo utilizando la metodología del estudio de casos (Rodríguez, 20I2; Stake, I999; Yin, 2009), aunque no se sigan de manera fidedigna las etapas de esta tipología (Bisquerra, 1989). La información se recoge mediante los instrumentos descritos tras varias intervenciones de los Terapiclowns entre los meses de febrero y abril. Estos payasos de hospital van un día a la semana al HUBU, los miércoles, y realizan la intervención personalizada dos payasos en cada habitación de la planta de Pediatría y en los servicios de Hospital de Día, UCI Pediátrica, Neonatos, Urgencias Pediátricas y Aula Hospitalaria. Su misión es contribuir en la mejora de la calidad de vida de los enfermos, sus familias y del personal sanitario, ayudándoles a soportar mejor su estancia en el hospital, y ofrecerles momentos de relax y distracción a través del humor, la risa y la fantasía.

Se administra el cuestionario a los niños en el Aula Hospitalaria en horario escolar, previo consentimiento firmado de sus padres y solicitud de datos sociodemográficos. Las entrevistas a los padres se realizan durante cuatro días alternos en un despacho privado dentro del Aula Hospitalaria, con permiso expreso para su grabación. En todos los casos fueron las madres quienes respondieron a las entrevistas.

\subsection{Análisis de datos}

Para el análisis de los datos procedentes de los cuestionarios se ha optado por aplicar estadísticos descriptivos y ordenar los resultados en tablas.

Teniendo en cuenta que los datos de las entrevistas son de tipo textual, se llevó a cabo un análisis de contenido. Los criterios utilizados para su análisis han sido: temáticos, considerando unidades en función del tema abordado tras localizar los segmentos que hablasen de un mismo tema o contenido afín; y gramaticales, diferenciado como unidades básicas del texto oraciones y párrafos al asumir que son unidades de información con sentido completo y similar contenido.

\section{Resultados}

\section{I. Características generales de la muestra}

Como se observa en la Tabla I, tres de los cuatro menores son niñas de 8 años con diferentes tipologías de leucemia. Todas ellas llevan en tratamiento oncológico 
USO DEL HUMOR COMO MÉTODO DE INTERVENCIÓN TERAPÉUTICA

EN MENORES AFECTADOS POR PROCESOS ONCOLÓGICOS

M. FERNÁNDEZ HAWRYLAK, D. HERAS SEVILLA, A. CEPA SERRANO Y J. BASTIDA SUSINOS

alrededor de un año (entre ıо y I3 meses). El otro menor que compone la muestra es un niño de 5 años que padece un neuroblastoma. En este caso, lleva en tratamiento 6 meses con hospitalizaciones de carácter breve, inferiores a la semana. Respecto a la modalidad de hospitalización, en todos los casos es diferente. Tan sólo el participante 3 (niña de 8 años) se encuentra en su domicilio. En los demás casos, se oscila entre una hospitalización breve y el ingreso hospitalario continuado. Como es habitual en las familias de menores con enfermedad crónica, las madres suelen ocupar el puesto de cuidador principal. En dos de los cuatro casos se da esta circunstancia. Es subrayable cómo en la familia del participante 2 los padres consideran que se reparten esta tarea. Respecto a las características del contexto familiar, en todos los casos los menores cuentan con un hermano o hermana de mayor edad. Finalmente, en cuanto a la escolarización de estos pequeños, se aprecia que las tres niñas de 8 años están cursando su curso de EPo en el Aula Hospitalaria, y el niño EI con apoyo tanto de su maestra del centro de referencia como de la del servicio de atención domiciliaria.

TABLA I. Resumen de las características generales de la muestra

\begin{tabular}{|c|c|c|c|c|c|c|c|c|c|}
\hline & Sexo & Edad & Patología & $\begin{array}{c}\text { Meses } \\
\text { de enfer- } \\
\text { medad }\end{array}$ & $\begin{array}{l}\text { Atención } \\
\text { sanitaria }\end{array}$ & $\begin{array}{l}\text { Cuidador } \\
\text { principal }\end{array}$ & $\begin{array}{l}\text { Tipo de } \\
\text { escola- } \\
\text { ridad } \\
\end{array}$ & $\begin{array}{c}\text { Curso } \\
\text { y etapa } \\
\text { educativa }\end{array}$ & $\begin{array}{c}\text { Herma- } \\
\text { nos/as }\end{array}$ \\
\hline $\begin{array}{l}\text { Sujeto } \\
\text { I }\end{array}$ & Varón & 5 & $\begin{array}{c}\text { Neuroblas- } \\
\text { toma }\end{array}$ & 6 meses & $\begin{array}{c}\text { Con } \\
\text { hospitalización } \\
\text { breve (menos de } \\
\text { una semana) }\end{array}$ & Madre & $\begin{array}{c}\text { Domici- } \\
\text { liaria }\end{array}$ & $3 .^{\circ} \mathrm{EI}$ & $\begin{array}{c}\text { Hermano } \\
\text { mayor }\end{array}$ \\
\hline $\begin{array}{l}\text { Sujeto } \\
2\end{array}$ & Mujer & 8 & $\begin{array}{l}\text { Leucemia } \\
\text { Linfoblás- } \\
\text { tica }\end{array}$ & Io meses & $\begin{array}{c}\text { Con } \\
\text { hospitalización } \\
\text { intermitente } \\
\text { (igual o superior } \\
\text { a una semana) } \\
\end{array}$ & Ambos & $\begin{array}{l}\text { Aula } \\
\text { Hospi- } \\
\text { talaria }\end{array}$ & $2{ }^{\circ} \mathrm{EPO}$ & $\begin{array}{c}\text { Hermana } \\
\text { mayor }\end{array}$ \\
\hline $\begin{array}{c}\text { Sujeto } \\
3\end{array}$ & Mujer & 8 & Leucemia & I2 meses & En el domicilio & Madre & $\begin{array}{c}\text { Aula } \\
\text { Hospi- } \\
\text { talaria }\end{array}$ & $2{ }^{\circ} \mathrm{EPO}$ & $\begin{array}{c}\text { Hermano } \\
\text { mayor }\end{array}$ \\
\hline $\begin{array}{c}\text { Sujeto } \\
4\end{array}$ & Mujer & 8 & Leucemia & I3 meses & Hospitalizado & $\mathrm{NS} / \mathrm{NC}$ & $\begin{array}{c}\text { Aula } \\
\text { Hospi- } \\
\text { talaria }\end{array}$ & $3 .^{\circ} \mathrm{EPO}$ & $\begin{array}{c}\text { Hermano } \\
\text { mayor }\end{array}$ \\
\hline
\end{tabular}

TABLA 2. Percepción infantil de los payasos en el hospital

\begin{tabular}{|l|c|c|c|}
\hline & $\begin{array}{c}\text { Nunca o pocas } \\
\text { veces (\%) }\end{array}$ & $\begin{array}{c}\text { Algunas } \\
\text { veces (\%) }\end{array}$ & $\begin{array}{c}\text { La mayoría } \\
\text { de las veces o } \\
\text { siempre (\%) }\end{array}$ \\
\hline La visita de los payasos de hospital: & & & \\
\hline $\begin{array}{l}\text {... la primera vez me hizo mucha ilusión, no } \\
\text { me lo esperaba. }\end{array}$ & 75.00 & & 25.00 \\
\hline ... hace que esté tranquila/o, relajada/o. & 50.00 & & 50.00 \\
\hline ... después de meses me hace ilusión. & 25.00 & & 75.00 \\
\hline ... me ayuda a distraerme y me siento bien. & & 25.00 & 75.00 \\
\hline ... me ayuda a afrontar la enfermedad. & 25.00 & & 75.00 \\
\hline
\end{tabular}




\subsection{Percepción infantil sobre los payasos en el hospital}

En relación a la percepción infantil sobre los payasos en el hospital (Tabla 2), se observa que al $75 \%$ de los menores no les hicieron especial ilusión las primeras visitas de los payasos, probablemente por el carácter novedoso y la propia asimilación de la enfermedad. Tan sólo un participante contestó que desde el primer momento «le hizo mucha ilusión la intervención de los payasos».

En la Tabla 2, se muestra también el diferente impacto de los Terapiclowns en la relajación y tranquilidad de los menores hospitalizados. De esta manera, el 50\% de los pequeños contestaron que siempre se encontraban relajados al ver a los payasos. En cambio, los otros dos menores consideraron que nunca o pocas veces se encontraban tranquilos con la visita de los payasos, muy probablemente porque dichas visitas les inclinaban al juego y al movimiento.

Las respuestas dadas en la escala breve evidencian que con el transcurso de la enfermedad y/o la progresiva adaptación a las rutinas hospitalarias, los menores valoran en mayor medida la intervención de los Terapiclowns. De esta manera, el 75\% de los pequeños considera que siempre o la mayoría de las veces les hace ilusión la visita de los payasos después de meses. Tan sólo un participante manifiesta no sentir ilusión al ver a los payasos después de meses. Cabe reseñar que es el paciente de menor edad y que además ha tenido menos tiempo para la aceptación y adaptación de su enfermedad.

En cuanto a la capacidad de los Terapiclowns para distraer a los pequeños y ayudar a su bienestar, se observa cómo el $75 \%$ de los menores hospitalizados percibe que los payasos les ayudan a distraerse y a sentirse bien siempre o la mayoría de las veces. Además, el menor restante considera que consiguen su distracción y bienestar algunas veces. Se puede entender, por tanto, que la labor de distracción de los payasos es efectiva y positiva para los menores.

Finalmente, se interrogó sobre la posible repercusión de la actividad de los payasos en el afrontamiento de la enfermedad. Nuevamente, el $75 \%$ de los pequeños entiende que siempre o la mayoría de las veces los payasos les ayudan a afrontar la enfermedad. En cambio, el sujeto I, que se halla en su sexto mes tras el diagnóstico, contestó que pocas veces le ayudan los payasos a afrontar la enfermedad; posiblemente, el afrontamiento estaba aún en proceso.

TABLa 3. Puntuación en la escala breve sobre percepción infantil de los payasos en el hospital

\begin{tabular}{|l|c|c|c|c|c|}
\hline & N & Mínimo & Máximo & Media & DT \\
\hline Puntuación de la muestra & 4 & 2.80 & 4.00 & 3.60 & .57 \\
\hline
\end{tabular}

Las puntuaciones globales en la escala evidencian la buena labor ejercida por los Terapiclowns, ya que la media entre todos los niños/as se sitúa en $3.6(D T=.57)$ en una escala con puntuaciones de i a 5 (Tabla 3). Si se analiza la puntuación de cada participante de forma independiente se observan resultados similares, pues hasta el menor de los participantes tiene una puntuación con una ligera tendencia positiva (Tabla 4). La edad y el tiempo hospitalizado parecen incidir en la menor puntación del sujeto I. No obstante, las otras tres niñas tienen una alta percepción y valoración de las visitas de los payasos. 
USO DEL HUMOR COMO MÉTODO DE INTERVENCIÓN TERAPÉUTICA

EN MENORES AFECTADOS POR PROCESOS ONCOLÓGICOS

M. FERNÁNDEZ HAWRYLAK, D. HERAS SEVILLA, A. CEPA SERRANO Y J. BASTIDA SUSINOS

TABla 4. Puntuación individualizada en la escala breve sobre percepción de los payasos

\begin{tabular}{|c|c|}
\hline Sujeto & Puntuación \\
\hline Sujeto I & 2.80 \\
\hline Sujeto 2 & 4.00 \\
\hline Sujeto 3 & 4.00 \\
\hline Sujeto 4 & 3.60 \\
\hline
\end{tabular}

\subsection{Resultados de las entrevistas}

Las entrevistas arrojaron una valiosa información sobre los principales efectos de los Terapiclowns en los menores y sus familias. Realizadas e impresas las transcripciones de cada una de las entrevistas, se efectuó una lectura exhaustiva, considerándose todos los elementos que en principio se descubrieron repetitivos, subrayando cada una de las palabras o frases consideradas significativas. Posteriormente, se elaboraron las categorías abiertas formadas por elementos coincidentes de la información ofrecida por las madres entrevistadas, reagrupando y relacionando las categorías con las subcategorías que fueron surgiendo. Se contrastaron uniformidades del conjunto de categorías y subcategorías, y se formuló una teoría que giró en torno a las respuestas de los niños hospitalizados y a las de sus padres ante los payasos del hospital y los efectos de estos últimos en el bienestar en general.

La primera categoría que se designó fue «Respuesta ante los payasos de hospital», agrupando las subcategorías en función de la reacción de los padres, la reacción inicial de los pacientes y la reacción de los participantes en el tiempo. La reacción ante los payasos de hospital tanto en los menores hospitalizados como en sus familias es positiva. Las madres entrevistadas expresaron que desconocían este servicio que ofrece la planta de Pediatría del HuBu, y que la primera vez que vieron a los Terapiclowns se quedaron sorprendidos y contentos, ya que, cuando reciben el diagnóstico, poder contar con este servicio les pareció importante, tal y como expresa una madre: «Vi que daban vida, alegría, en un primer momento reaccioné con alegría, porque después de recibir la noticia, pues un soplo de alegría nunca viene mal» (Madre sujeto 4). Además, aunque algunos de los pequeños al principio mostraron indiferencia, al cabo de los meses les hace mucha ilusión y les ayuda a sentirse bien: «Ahora sí, le hace mucha ilusión, ya les conoce, se divierte con ellos» (Madre sujeto 2).

La segunda categoría designada fue «Beneficios de los Terapiclowns en el afrontamiento de la enfermedad», desde la que se agruparon las subcategorías sobre la mejora física, la mejora psicológica y la mejora familiar. Las madres entrevistadas opinaron que los payasos no mejoran el tratamiento de la enfermedad, pero sí influyeron positivamente en el estado de ánimo y en la actitud de los pacientes durante la hospitalización, como puede leerse en el testimonio de otra de las madres: «¡Pues hombre! Que haya influido a mejorar el tratamiento... pues bueno, es lo que es y no es que influya, pero sí a lo mejor en su actitud a la hora de venir» (Madre sujeto I). Además también benefician y mejoran la cohesión familiar, según manifestaba una madre a propósito de la comunicación: «Emotivo para mí y para ella, porque cuando ellos se van, su conversación son los payasos» (Madre sujeto 3). Igualmente es positivo para las familias que informan reírse también y pasarlo bien, como afirmaba esta misma madre: "Yo no 
me acordaba casi, ni que estaba en el hospital, me estaba riendo y lloraba y yo diciendo es que estoy llorando de risa, de lo bien que me lo estoy pasando» (Madre sujeto 3).

\section{Discusión y conclusiones}

El estudio muestra que el uso del humor a través de los payasos de hospital puede cambiar la forma de abordar la enfermedad. A la vista de estos resultados se puede afirmar que la intervención de los Terapiclowns es recomendable y positiva.

En relación a los beneficios, la actuación de los Terapiclowns en niños afectados por procesos oncológicos reporta ventajas, como es la mejora del estado de ánimo al proporcionar una zona segura, «mágica», donde las exigencias de la enfermedad se apartan temporalmente, y donde un lado más amable de la vida aflora, siendo la perspectiva del niño el centro de atención. También distraen o desactivan la atención de la situación de sufrimiento derivada de la enfermedad y de la hospitalización, al utilizar actuaciones distintas y lúdicas ante las rutinas regulares del hospital, haciéndolas más fáciles para los pequeños y para sus padres.

Los estudios de casos se basan en la recopilación de información detallada sobre una persona o grupo a lo largo de un tiempo considerable. El estudio avala el uso del humor como técnica activa del afrontamiento eficaz de la enfermedad, aun siendo limitado en el tiempo, y necesaria una investigación más pormenorizada sobre la intervención de los payasos de hospital en los diferentes momentos de la hospitalización de menores con enfermedades oncológicas o afectados por otro tipo de enfermedades de larga duración, como podrían ser las enfermedades minoritarias. Los resultados encontrados en este estudio ponen de relieve la necesidad de estudiar en profundidad diferentes modelos de inclusión de la terapia con payasos, así como los efectos de la misma en las distintas fases de aceptación de la enfermedad. Incluso, se podría plantear estudiar la introducción del humor en niños afectados por enfermedades de larga duración como herramienta para lograr aprendizajes, esto es, dentro del campo de la Pedagogía Hospitalaria.

Partiendo de las preguntas de investigación planteadas, las principales conclusiones del estudio realizado son: el uso del humor en niños con procesos oncológicos ayuda a afrontar mejor la enfermedad; las reacciones de los niños hospitalizados ante los payasos de hospital son positivas; la presencia de los payasos en el hospital proporciona beneficios importantes, como la mejora en el estado de ánimo de los niños y de sus padres; la intervención de los payasos de hospital contribuye a desarrollar una actitud más positiva hacia el tratamiento y las pruebas médicas; el uso del humor en niños con enfermedades oncológicas es una técnica activa de afrontamiento de la enfermedad.

\section{Bibliografía}

AECC (s. f.). El cáncer en los niños. Barcelona: Autor.

Attardo, S.; Hempelmann, C. F. y Di Maio, S. (2002) Script oppositions and logical mechanisms: Modeling incongruities and their resolutions. Humor International Journal of Humor Research, I5 (I), 3-46. doi:10.1515/humr.2002.004. 
USO DEL HUMOR COMO MÉTODO DE INTERVENCIÓN TERAPÉUTICA

EN MENORES AFECTADOS POR PROCESOS ONCOLÓGICOS

M. FERNÁNDEZ HAWRYLAK, D. HERAS SEVILLA, A. CEPA SERRANO Y J. BASTIDA SUSINOS

Baliari, B. y Rosado, T. (20Io) Lo esencial es invisible a los ojos: payasos que humanizan y promueven salud. Aletheia Revista de Psicologia, 3I, 4-I5.

BALICK, M. y LEE, R. (2003) The role of laughter in traditional medicine and its relevance to the clinical setting: Healing with ha! Alternative Therapies in Health and Medicine, 9 (4), 88-9I.

Barkmann, C.; Siem, A. K.; Wessolowski, N. y Schulte-Markwort, M. (20i3) Clowning as a supportive measure in paediatrics - a survey of clowns, parents and nursing staff. $B M C$ Pediatrics, I3, I66. doi:IO.II86/I47I-243I-I3-I66.

Battrick, C.; Glasper, E. A.; Prudhoe, G. y Weaver, K. (2007) Clown humour: the perceptions of doctors, nurses, parents, and children. Journal of Children's and Young People's Nursing, I (4), I74-179.

Benito, A. y García, J. (2013) Terapiclowns: un proyecto innovador aplicado a la rehabilitación de personas con enfermedad mental grave. PsiQuiatría-Ciencia, Arte y Humanidades, $6(3), 8-13$.

Berk, R. A. (200I) The active ingredients in humor: Psychophysiological benefits and risks for older adults. Educational Gerontology, 27, 323-39. doi:10.1080/036012701750195021.

Billing, M. (2002) Freud and the language of humour. The Psychologist, 15, 452-455.

Bisquerra, R. (1989) Métodos de investigación educativa. Barcelona: CEAc.

Camuñas, A. (2009) El teatro clown en el entorno sanitario. Index de Enfermería, i8 (I), 63-65.

Capps, D. (2006) The psychological benefits of humor. Pastoral Psychology, 54 (5), 393-4II. doi:Io.1007/siro89-005-0007-9.

CARbelo, B. (2005) El Humor en la relación con el paciente: una guía para profesionales de la salud. Barcelona: Elsevier.

Carbelo, B. y Jáuregui, E. (2006) Emociones positivas: humor positivo. Papeles del Psicólogo, 27 (I), I8-30.

Christian, R.; Ramos, J.; Susanibar, C. y Balarezo, G. (2004) Risoterapia: Un nuevo campo para los profesionales de la salud. Revista de la Sociedad Peruana de Medicina Interna, 17 (2), 57-64.

De la Gándara, J. (2013) El buen humor y la salud mental. PsiQuiatría-Ciencia, Arte y Humanidades, 6 (3), 6-7.

ERICKSON, S. y FeLDSTEIn, S. (2007) Adolescent humor and its relationship to coping, defense strategies, psychological distress, and well-being. Child Psychiatry and Human Development, 37 (3), 255-27I. doi:10.1007/sios78-006-0034-5.

Espada, M. ${ }^{a}$ C.; Grau, C. y Fortes, M. ${ }^{a}$ C. (2OIO) Enseñar estrategias de afrontamiento a padres de niños con cáncer a través de un cortometraje. Anales del Sistema Sanitario de Navarra, 33 (3), 259-269.

FEPNC (2007) Psico-oncología pediátrica: valoración e intervención. Barcelona: Autor.

FEPNC (20I4) Cáncer en menores. Recuperado el I2 de abril de 20I6, de http://cancerinfantil.org/.

Fernández-Abascal, E. G. (coord.) (2009) Emociones positivas. Madrid: Pirámide.

Forabosco, G. (1992) Cognitive aspects of the humor process: the concept of incongruity. Humor-International Journal of Humor Research, 5 (I-2), 45-68. doi:Io.I5I5/humr.1992.5.I-2.45.

Ford, T. E. y Ferguson, M. A. (2004) Social Consequences of Disparagement Humor: A Prejudiced Norm Theory. Personality E Social Psychology Review, 8 (I), 79-94. doi:Io.I207/ Sis327957PSPRo8oI_4.

Forman, D.; Bray, F.; Brewster, D. H.; Gombe-Mbalawa, C.; Kohler, B.; Piñeros, M. y Steliarova-Foucher (eds.) (20I4) Cancer Incidence in Five Continents, vol. x. IARC Scientific Publication, i64. Recuperado de http://www.iarc.fr/en/publications/pdfs-online/ epi/spi64/CI5volX_Full.pdf.

FRY, W. F. (2002) Humor and the brain: a selective review. Humor-International Journal of Humor Research, I5 (3), 305-333. doi:10.1515/humr.2002.017.

Gruner, C. R. (1997) The game of humor: a comprehensive theory of why we laugh. New Brunswick, NJ: Transaction. 
Gutiérrez, M. A.; Ortigosa, J. M.; Girón, O.; Ruiz, R.; Sánchez, J.; Guirao, M. J. y Zambudio, G. (2008) Evaluación del efecto de la actuación de los payasos de hospital sobre la ansiedad, en los niños sometidos a una intervención quirúrgica. Cirugía Pediátrica, 2I (4), I95-I98.

Instituto Gesundheit (s. f.) Recuperado el i4 de abril de 20I6, de http://www.patchadams.org/. Joubert, L. (1579/2002) Tratado de la risa. Madrid: Asociación Española de Neuropsiquiatría.

Klein, A. (2003) Introduction: A global perspective of humor. En A. Klein (ed.) Humor in children's lives: A guidebook for practitioners (pp. 3-15). Westport: Preager Publishers.

Koller, D. y Gryski, C. (2008) The Life Threatened Child and the Life Enhancing Clown: Towards a Model of Therapeutic Clowning. Evidence-Based Complementary and Alternative Medicine, 5 (I), 17-25. doi:I0.1093/ecam/nemo33.

Kuiper, N.; Grimshaw, M.; Leite, C. y Lirsh, G. (2004) Humor is not always the best medicine: Specific components of sense of humor and psychological well-being. Humor-International Journal of Humor Research, 17 (I/2), I35-I68. doi:IO.I5I5/humr.2004.002

LiNGE, L. (2013) Joyful and serious intentions in the work of hospital clowns: A meta-analysis based on a 7-year research project conducted in three parts. International Journal of Qualitative Studies on Health and Well-Being, 8, 18907. doi:10.3402/qhw.v8io.I8907

Martin, R. A. (2000) Humor and Laughter. En A. E. Kazdin (ed.) Encyclopedia of Psychology, vol. 4. New York: Oxford University Press.

Martin, R. A. (200I) Humor, laughter, and physical health: Methodological issues and research findings. Psychological Bulletin, I27 (4), 504-519. doi:I0.1037//0033-2909.127.4.504.

Martin, R. A. (2004) Sense of humor and physical health: Theoretical issues, recent findings, and future directions. Humor: International Journal of Humor Research, I7 (I-2), I-I9. doi:I0.1515/humr.2004.005.

Martin, R. A.; Puhlik-Doris, P.; Larsen, W.; Gray, J. y Weir, K. (2003) Individual differences in uses of humor and their relation to psychological well-being: Development of the Humor Styles Questionnaire. Journal of Research in Personality, 37 (I), 48-75. doi:Io.Ior6/ Soo92-6566(02)00534-2.

Meisel, V.; Chellew K.; Ponsell, E.; Ferreira, A.; Bordas, L. y García-Bandas, G. (2009) El efecto de los «payasos de hospital» en el malestar psicológico y las conductas desadaptativas de niños y niñas sometidos a cirugía menor. Psicothema, 2I (4), 604-609.

Moliner, M. (20oI) Diccionario de uso del español (2. ${ }^{\mathrm{a}}$ ed., 3. ${ }^{\mathrm{a}}$ reimp.). Madrid: Gredos.

Mora, R. y Quintana, I. (20I0) Risa y terapias positivas: moderno enfoque y aplicaciones prácticas en medicina. Revista de Psiquiatría y Salud Mental, 3 (I), 27-34. doi:Io.IoI6/SI888989I(Io)70006-9.

Norrick, N. R. (2003) Issues in conversational joking. Journal of pragmatics, 35 (9), 1333-1359. doi:Io.1016/So378-2166(02)oor8o-7.

Perlmutter, D. D. (2000) Tracing the origin of humor. Humor-International Journal of Humor Research, I3 (4), 457-468. doi:10.1515/humr.2000.13.4.457.

Provine, R. R. (2004) Laughing, Tickling, and the Evolution of Speech and Self. Current Directions in Psychological Science, I3 (6), 215-218. doi: I0.1111/j.0963-7214.2004.00311.x.

Roeckelein, J. (2002) The Psychology of Humor: A Reference Guide and Annotated Bibliography. London: Greenwood Press.

Rodríguez, M. (20I2) Cómo investigar con Estudios de Casos. Bolivia: CEdid-FIFIEd.

Rodríguez, G.; Gil, J. y García, E. (1999) Metodología de la investigación cualitativa, 2. a ed. Archidona: Aljibe.

Ruch, W. (1998) Foreword and overview. Sense of humor: A new look at an old concept. En W. Ruch (ed.) The Sense of Humor. Explorations of a Personality Characteristic (pp. 3-I4). Berlín: de Gruyter Mouton.

Ruiz, C.; Rojo, C.; Ferrer, A.; Jiménez, L. y Ballesteros, M. (2005) Terapias complementarias en los cuidados. Humor y risoterapia. Index Enfermermería, I4 (48-49), 37-4I. 
USO DEL HUMOR COMO MÉTODO DE INTERVENCIÓN TERAPÉUTICA

EN MENORES AFECTADOS POR PROCESOS ONCOLÓGICOS

M. FERNÁNDEZ HAWRYLAK, D. HERAS SEVILLA, A. CEPA SERRANO Y J. BASTIDA SUSINOS

SÁnchez, J.; Gutiérrez, J.; Santacruz, J.; Romero, C. y Ospina, J. (2009) El humor como estrategia terapéutica en niños hospitalizados en unidades pediátricas en Pereira (Colombia). Reporte de una experiencia. Revista Colombiana de Psiquiatría, 38 (I), 99-II3.

StAKe, R. (1999) Investigación con estudios de casos. Madrid: Morata.

Sultanoff, S. (1999) Examining the Research on Humor: Being Cautious About Our Conclusions. Therapeutic Humor, I2 (3), 3.

Torres, H. (2003) El humor como herramienta para lograr aprendizajes. Telos-Revista de Estudios Interdisciplinarios en Ciencias Sociales, 5 (3), 408-423.

Wycoff, E. B. y Pryor, B. (2003) Cognitive Processing, Creativity, Apprehension \& the Humorous Personality. North American Journal of Psychology, 5 (I), 3I-44.

YIn, R. (2009) Case Study Research. Design and Methods (4. ${ }^{\text {a }}$ ed.). London: SAgE. 